\title{
Pharmacogenomics and personalized medicine: the plunge into next-generation sequencing
}

\author{
Mia Wadelius ${ }^{1 *}$ and Ana Alfirevic ${ }^{2}$
}

\begin{abstract}
A report on the 9th Annual Cold Spring Harbor/

Wellcome Trust meeting 'Pharmacogenomics and

Personalized Medicine', Hinxton, Cambridge, UK,

29 September to 2 October 2011.

Keywords Consortium, genome-wide association study, meta-analysis, next-generation sequencing, pharmacogenomics/pharmacogenetics, public health genomics, P4 medicine.
\end{abstract}

In recent years, pharmacogenomics has moved beyond candidate gene and genome-wide association studies (GWASs) towards truly personalized genomics. The use of new biotechnological, mathematical and computational tools has enabled an exponential increase in the number of biomarkers for drug safety and efficacy; however, their clinical utility in achieving personalized therapy remains to be determined. Here we cover current expert opinions concerning emerging pharmacogenomic technologies, international consortia and collaborations including underrepresented populations, development of personalized medicine and clinical relevance of pharmacogenetic testing. In addition, future directives presented at the meeting are discussed.

\section{Technology-driven research}

Since the first Wellcome Trust/Cold Spring Harbor Laboratory meeting on pharmacogenetics in 2003, the field has evolved into pharmacogenomics through a shift from candidate gene studies to GWASs. Such large-scale studies enable simultaneous detection of $>1$ million SNPs that can be tested for association with drug-related outcomes, and verified by replication in separate cohorts. The development of next-generation sequencing (NGS)

\footnotetext{
*Correspondence: mia.wadelius@medsci.uu.se

'Department of Medical Sciences, Clinical Pharmacology, Uppsala University,

Uppsala University Hospital, entrance 61, SE-751 85 Uppsala, Sweden

Full list of author information is available at the end of the article
}

technology has led to a drastic drop in the cost $(>10,000$-fold) and time (from 10 years to 1 week) needed to sequence a genome. NGS is now being introduced as a method to personalize medicine.

Yingrui Li (Beijing Genomics Institute, China) described the sequencing revolution that has made personal genomes affordable, while it remains difficult and costly to interpret the results. Recent whole genome sequencing of individuals at the Beijing Genomics Institute has shown an excess of rare deleterious SNPs together with extensive structural variations and novel individual specific sequences with potential functional impact. These new genetic variants are likely to explain part of the missing heritability seen with previous GWASs. Interestingly, Li reported that more ethnic-specific haplotypes exist than previously thought and that each defined population will need its own genome-wide tag SNP array. This is a general problem that must be considered when performing pharmacogenetic GWASs in non-Caucasian populations.

Steve Scherer (The Hospital for Sick Children, Canada) shared his enthusiasm over large-scale sequencing efforts within the Pharmacogenomics Research Network (PGRN) Deep Sequencing Resource (DSR). This network is supported by the National Institutes of Health (NIH), and consists of 14 US research groups. Ongoing projects are: targeted re-sequencing in pharmacogenetic breast cancer and hypertension studies, platelet RNA sequencing in responders and non-responders to clopidogrel, and RNA sequencing in tissues of major pharmacologic interest to assess gene expression levels of common splice variants in very important pharmacogenes (VIPs). PGRN DSR is also developing a gene capture reagent for sequencing of VIPs, such as cytochrome P450 genes and human leukocyte antigen (HLA) genes. Scherer concluded that these large-scale sequencing projects have the potential to transform medicine, but some important issues, such as the generation of software to present results to physicians in a coherent manner, remain to be solved.

\section{Meta-analyses and consortia}

The ability of high-throughput genotyping technologies to interrogate millions of markers simultaneously in 


\begin{tabular}{|c|c|c|c|}
\hline Group & Abbreviation & Website & Presenter(s) \\
\hline Breast Cancer Association Consortium & BCAC & http://www.srl.cam.ac.uk/consortia/bcac/ & Christina Justenhoven \\
\hline Canadian Pharmacogenomics Network for Drug Safety & CPNDS & http://www.cpnds.ubc.ca/ & $\begin{array}{l}\text { Michael Hayden, Colin Ross, } \\
\text { Ursula Amstutz }\end{array}$ \\
\hline Clinical Pharmacogenetics Implementation Consortium & CPIC & $\begin{array}{l}\text { http://www.pharmgkb.org/contributors/ } \\
\text { consortia/cpic_profile.jsp }\end{array}$ & Ellen M McDonagh \\
\hline Human Heredity and Health in Africa Initiative & H3Africa & http://h3africa.org/ & Collet Dandara \\
\hline Inflammation and the Host Response to Injury Consortium & IHRI & https://www.gluegrant.org/index.htm & Wenzhong Xiao \\
\hline International Cancer Genome Consortium & ICGC & http://www.icgc.org/ & Ultan McDermott \\
\hline International Serious Adverse Events Consortium & ISAEC & http://www.saeconsortium.org/ & Ann Daly \\
\hline International Warfarin Pharmacogenetics Consortium & IWPC & $\begin{array}{l}\text { http://www.pharmgkb.org/contributors/ } \\
\text { consortia/iwpc_profile.jsp }\end{array}$ & Stephane Bourgeois \\
\hline Pharmacogenomics Research Network & PGRN & http://pgrn.org/ & Steven Scherer \\
\hline SpiroMeta Consortium & - & - & Ma'en F Obeidat \\
\hline
\end{tabular}

association studies necessitates the use of large sample sizes that can be recruited only through collaborations; some studies reported at the meeting included $>90,000$ participants. The trend is therefore to perform metaanalyses of available GWASs to increase statistical power, and also to persuade old competitors to work together in consortia (Table 1), where primary individualized outcome data are pooled with previous or new genotyping data. This is indeed a positive effect brought on by recent technological developments.

Several consortia are hosted by the PharmacoGenomics Knowledge Base (PharmGKB). Among them is the Clinical Pharmacogenetics Implementation Consortium, which is aimed at providing guidance on how to adjust medications on the basis of pharmacogenetic test results. Another PharmGKB network is the International Warfarin Pharmacogenetics Consortium, which was intended initially for the development of a warfarin dose algorithm, but is now involved in a warfarin GWAS meta-analysis. Two consortia aimed at investigating genetic risk factors for adverse drug reactions were presented. First, Ann Daly (Newcastle University, UK) from the International Serious Adverse Events Consortium described recent results concerning GWASs and exome sequencing of serious adverse drug reactions affecting the liver. Thereafter, Michael R Hayden, Colin Ross and Ursula Amstutz (University of British Columbia, Canada) presented studies of severe adverse drug reactions in children within the Canadian Pharmacogenomics Network for Drug Safety. The International Cancer Genome Consortium, which is searching for biomarkers of sensitivity and resistance to therapies by exposing 1,000 cancer cell lines to a multitude of chemical compounds, was described by Ultan McDermott (Wellcome Trust Sanger Institute, UK). Finally, Christina Justenhoven (Dr Margarete FischerBosch-Institute of Clinical Pharmacology, Germany) represented the Breast Cancer Association Consortium, which is searching for breast cancer susceptibility genes, as well as studying effects of hormone therapy in relation to genotypes. One main driver of these networks (except the Clinical Pharmacogenetics Implementation Consortium) is to pool enough samples to enable robust study design with high statistical power.

\section{Pharmacogenomics in Africa}

African researchers and populations are substantially under-represented in most international research networks. It was therefore a pleasure to hear Collet Dandara (University of Cape Town, South Africa) describe the Human Heredity and Health in Africa (H3Africa) initiative, which is sponsored by the NIH and the Wellcome Trust. Specific issues in African populations are the great genetic diversity and the fact that most drugs are developed for non-Africans. Drug safety and efficacy biomarkers that have already been discovered may not be appropriate in African populations because of different allele and haplotype frequencies. Recent studies have shown that HLA types that confer risk of serious skin reactions vary between different populations. Hence it is essential to develop diagnostic and prognostic tools for all populations. Folefac Aminkeng (University of British Columbia, Canada) described an important project aimed at studying the pharmacogenetics of World Health Organization essential medicines in Africa to enable the most effective use of available medical resources, and Marelize Swart (University of Cape Town, South Africa) illustrated this endeavor by describing pharmacogenetic predictors of response to HIV/AIDS treatment.

\section{Clinical validity and utility of pharmacogenetic tests}

Numerous pharmacogenetic biomarkers for drug safety and efficacy have been identified over the past few years. 


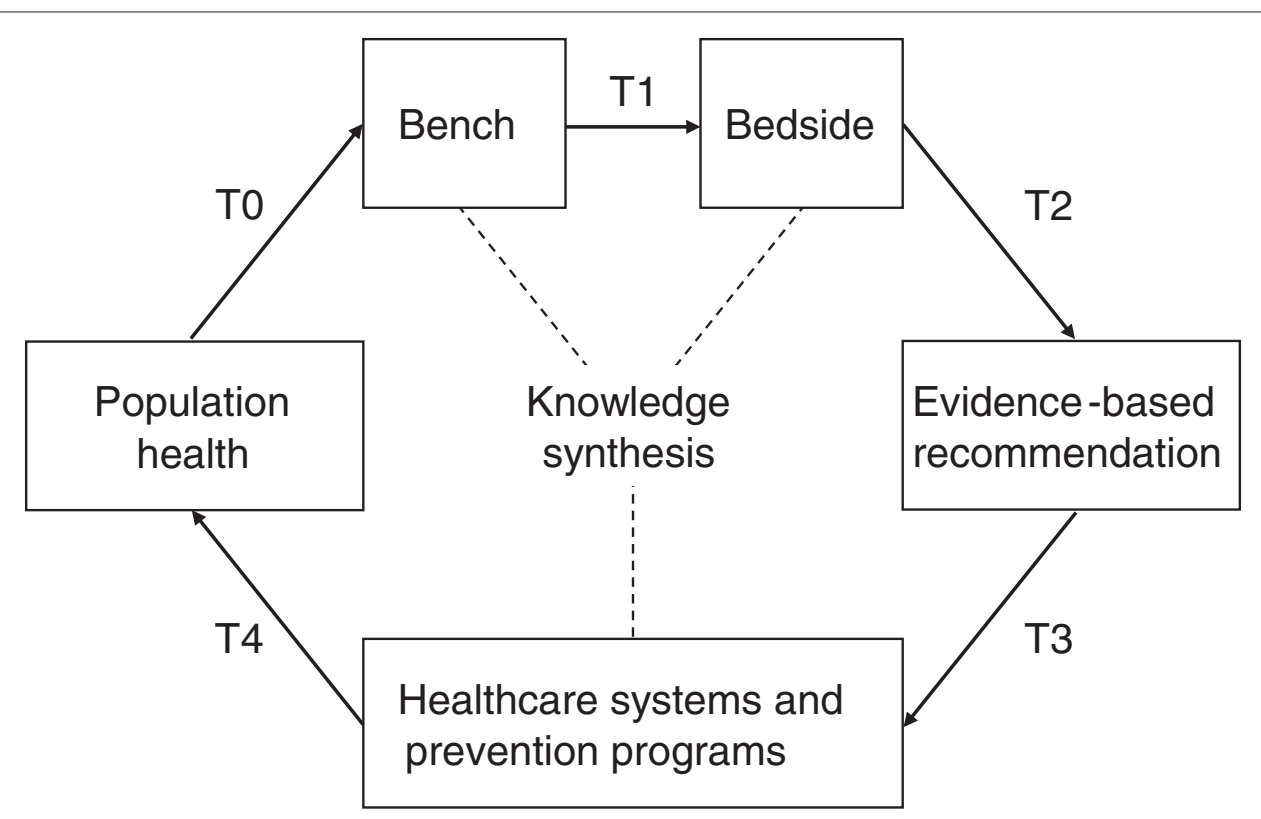

Figure 1. The genomics translation (T) highway. This model describes the translation from bench to bedside, and the knowledge that is synthesized from all parts of the process: T0, public health drives discovery; $\mathrm{T} 1$, discovery to application; $\mathrm{T} 2$, application to guideline; $\mathrm{T} 3$, guideline to practice; and T4, practice to population health impact. This figure has been modified and reproduced with the permission of Muin J Khoury.

At this meeting, biomarkers relating to a wide range of drugs were presented: from anticoagulants to immunotherapy, chemotherapy, antiretroviral therapy, antiepileptic drugs and antibiotics. The US Food and Drug Administration has currently more than 70 black box warnings for these drug-gene interactions. The European Medicines Agency has been considerably more conservative in this respect. The fact that only a few pharmacogenetic biomarkers have reached the clinic was touched upon by several speakers, most notably Muin J Koury (Office of Public Health Genomics, USA). Koury defined the translation process from genomic research to clinical and public health interventions as a cycle of five phases: T0 to T4 (Figure 1). Although the first phase of translation has acceptable levels of funding, subsequent phases are comparatively under-resourced and lack the necessary infrastructure; thus, priority should be given to these areas of research. After his presentation, the question of whether genetic biomarkers for drug response need the same level of evaluation as biomarkers for disease risk was raised.

This discussion was continued when for the first time in the Cold Spring Harbor Laboratory/Wellcome Trust series of pharmacogenomics meetings, a debate took place. It was led by Hiltrud Brauch ( $\mathrm{Dr}$ Margarete Fischer-Bosch-Institute of Clinical Pharmacology, Germany) and Paul Pharoah (Cambridge Cancer Centre, UK). The question set was: does CYP2D6 genotyping for response to tamoxifen treatment of estrogen-positive breast cancer have clinical validity and utility? Brauch presented arguments for, and Pharoah against, genotyping of CYP2D6. The issue was not settled here, but it initiated a heated discussion that was very well received by the contributing audience. In reality, the uptake of this and many other pharmacogenetic tests into the clinic has been slow because of the lack of evidence for clinical validity and utility. Concentrated efforts should be made to provide this evidence before pharmacogenetic tests are translated on a broad scale into clinical recommendations and policies.

\section{Beyond genotyping: P4 medicine}

Despite current reluctance to adopt pharmacogenetic testing, Leroy Hood (Institute of Systems Biology, USA) predicts that in 10 years time each patient will be surrounded by billions of data points: DNA sequence, imaging and test results, and so on. However, most of these data points will be biological noise, and how to select the relevant data, integrate them and formulate them into models will be the key to the future. The challenge will be to deal with this incredible complexity to decipher biological pathways implicated in disease and response to drugs. Hood described how emerging biotechnological, computational and mathematical tools will enable medicine to focus more on health (wellness) than disease. $\mathrm{He}$ calls the transition from reactive to proactive medicine $\mathrm{P} 4$ medicine, which is the term coined for predictive, preventive, personalized and participatory medicine. This is clearly an area of research for the future. 


\section{Future perspective}

Pharmcogenomic research is about to take the plunge into NGS. This technology, together with international collaborations to achieve large sample sizes, will surely be fruitful for pharmacogenetic research. Many biomarkers for drug safety and efficacy have been detected already, and we expect the number to increase over the next few years. However, as the proof of clinical validity and utility is lacking, only a few pharmacogenetic biomarkers have reached the clinic. It is important that not only Caucasians, but all diverse ethnicities across the world, are included in the process. The translation of pharmacogenomic biomarkers into the clinic will therefore be a greater endeavor than their discovery. We will undoubtedly hear more about this at future meetings. Given the complexity of integrating medicine with technology and computation through a systems biology approach, we may have to refine models to explain biological function at several levels before personalized medicine is a reality. The ability of the international scientific community to tackle complexity exists and was clearly demonstrated at the meeting.

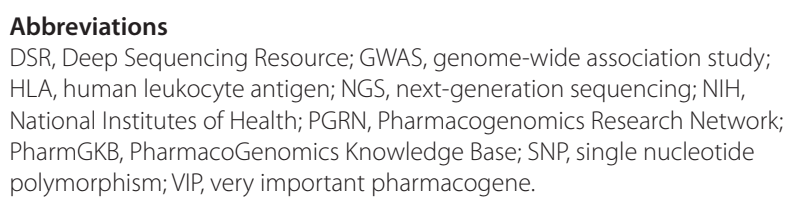

\section{Competing interests}

The authors declare that they have no competing interests.

Authors' contributions

Both authors have been involved in drafting the manuscript and revising it critically, and have given final approval of the version to be published.

\section{Acknowledgements}

We thank Muin J Khoury for permission to modify and reproduce his flowchart. MW is supported by the Swedish Research Council (Medicine 523-2008-5568), the Swedish Heart and Lung Foundation, EU FP7 (HEALTH-F2-2009-223062) and the Clinical Research Support (ALF) at Uppsala University. The funding organizations played no role in the writing of this meeting report.

\section{Author details}

'Department of Medical Sciences, Clinical Pharmacology, Uppsala University, Uppsala University Hospital, entrance 61, SE-751 85 Uppsala, Sweden. ${ }^{2}$ The

Wolfson Centre for Personalised Medicine, Department of Molecular and Clinical Pharmacology, Institute of Translational Medicine, University of Liverpool, Block A: Waterhouse Buildings, 1-5 Brownlow Street, Liverpool L69 3GL, UK.

Published: 28 December 2011
Cite this article as: Wadelius M, Alfirevic A: Pharmacogenomics and personalized medicine: the plunge into next-generation sequencing. Genome Medicine 2011, 3:78. 